\title{
The Outcome Of Indonesian Protocol Chemotherapy Acute Lymphoblastic Leukemia 2018 Induction Phase at Dr. Soetomo Hospital
}

\author{
Muhammad Shofi Rafsyanjani ${ }_{2}^{1}$ I Dewa Gede Ugrasena ${ }^{1}$, \\ Mia Ratwita Andarsini ${ }^{1}$, Maria Christina Shanti Larasanti ${ }^{1}$, Andi Cahyadi ${ }^{1}$ \\ official email address: shofirafsyanjani@gmail.com
}

1Child Health Department, Medical Faculty, Airlangga University/Dr. Soetomo General Hospital, Surabaya 60286-Indonesia

2Clinical Pathology Department, Medical Faculty, Airlangga University/Dr. Soetomo General Hospital, Surabaya 60286-Indonesia

\begin{abstract}
Background: The prognosis of pediatric acute lymphoblastic leukemia (ALL) has improved in the last 50 years. In Indonesia, national protocol of ALL chemotherapy is divided into 2 risk stratification, standard risk and high risk. The outcome after the induction phase of chemotherapy has an important role and has prognostic factor for the success of ALL chemotherapy. This study aim to determine the outcome of induction phase chemotherapy using the pediatric ALL 2018 national protocol at Dr. Soetomo Hospital.
\end{abstract}

Methods: This prospective study was conducted from November 2020 to May 2021. Consecutive non-random sampling was done to children aged 1-18 years, recently diagnosed with ALL, and met the inclusion-exclusion criteria. Risk stratification was made based on age, initial leukocyte count, presence of a mediastinal mass, presence of CNS leukemia, and immunophenotyping. Evaluation of bone marrow aspiration was done after the sample had completed the induction phase of ALL chemotherapy according to the 2018 ALL national protocol stratification.

Results: Twenty-one children recently diagnosed with ALL and underwent chemotherapy. Fourteen children underwent standard risk national protocol of ALL chemotherapy, and 7 remaining with high risk. Fifteen children completed the induction phase of chemotherapy, 6 remaining children died before completing. All patient underwent standard risk chemotherapy can complete induction phase. All patients who completed the induction phase showed remission on bone marrow aspiration evaluation.

Conclusion: Patients who underwent standard risk of Indonesian protocol chemotherapy ALL 2018 have better outcome than high risk.

Keywords: ALL; leukemia; p53; chemotherapy; pediatric 


\section{Introduction}

The prognosis of pediatric acute lymphoblastic leukemia (ALL) has improved in the last 50 years with a remission rate up to $90 \%$ [1]. At Dr. Soetomo Hospital in 2013-2014, using the 2013 Indonesian ALL chemotherapy protocol, the outcome of post-induction chemotherapy remission only occurred in $46.4 \%$ of 143 children diagnosed with ALL. Chemotherapy remission in ALL with standard risk is $33.9 \%$ and high risk is $17.8 \%$ with a 3 -year free survival rate of $43-56 \%$ [2]. Failure of chemotherapy is influenced by many factors [3]. ALL chemotherapy in Indonesia is divided into 2 protocol, standard risk and high risk. The induction phase is the first phase of chemotherapy, aiming to eradicate cancer cells in order to achieve remission (9598\%). The outcome of chemotherapy after the induction phase has an important role and prognostic factor for the success of chemotherapy for ALL [2]. Therefore, the aim of this study was to determine the outcome of induction phase chemotherapy using the pediatric ALL 2018 national protocol at Dr. Soetomo Hospital.

\section{Subjects and Methods}

This study is a prospective observational study to determine the outcome of induction phase chemotherapy using the pediatric ALL 2018 national protocol at Dr. Soetomo Hospital. This study was conducted from November 2020 to May 2021 in Dr. Soetomo General Academic Hospital. This study was granted letter of exemption by the Ethic Committee of Dr. Soetomo General Academic Hospital Ref. No.: 0116/LOE/301.4.2/IX/2020. The inclusion criteria for this study were children aged 1-18 years, newly diagnosed with LLA who underwent chemotherapy at Dr. Soetomo General Academic Hospital, and their parents were willing to participate in the research. Children with LLA-L3 morphology and underwent chemotherapy outside Dr. Soetomo General Academic Hospital were excluded.

The diagnosis of leukemia is established based on history, physical examination, laboratory and bone marrow aspiration. Risk stratification chemotherapy is chosen if there are no signs of high risk ALL. Children will be treated with high-risk ALL if at the time of diagnosis one of the following criteria is obtained: age less than 1 year or more than 10 years, leukocytes more than 50,000x109/L, mediastinal mass more than two-thirds of the diameter of the chest cavity, there are more of 15/3 $(5 \mu \mathrm{m})$ leukemic cells in cerebrospinal fluid, and leukemia T-cell by immunophenotyping.

Evaluation of bone marrow aspiration was carried out after the sample had completed the induction phase of ALL chemotherapy according to the 2018 ALL national protocol stratification. Outcome was assessed by calculating the percentage of lymphoblast in bone marrow aspirate smears. Remission was defined as the lymphoblast count in the bone marrow blood smear after the induction phase of chemotherapy is less than $5 \%$. Data on patients who lived and died after the induction phase were also recorded as outcomes.

Collected data were analyze using Statistical Product and Service Solution (SPSS) version 25.0. Univariate analysis was done to describe the characteristics of the study subjects listed above.

\section{Results}

A total of 21 children were newly diagnosed with ALL underwent chemotherapy. Boys are more dominant than girls. The majority of the subjects' age ranged from 1-10 years with a median age of 6.4 years. Subjects' characteristic are shown in Table 1.

Table 1. Subjects' characteristics

Characteristics $\quad$ Frequency




\begin{tabular}{ll}
\hline & $\mathrm{n}(\%)$ \\
\hline Sex & \\
- Boy & $16(76,2)$ \\
- Girl & $5(23,8)$ \\
Age & \\
$-1-10$ year & $18(85,7)$ \\
$->10$ year & $3(14,3)$ \\
\hline
\end{tabular}

Symptoms and signs vary from child to child. In this study, the most common symptom was fever and the most common sign was hepatomegaly. Symptoms and signs of subject are shown in table 2.

Table 2. Subjects' symptoms and signs

\begin{tabular}{ll}
\hline Symptoms and signs & Frequency \\
& $\mathrm{n}(\%)$ \\
\hline Pale & $16(76,2)$ \\
Bleeding & $10(47,6)$ \\
Fever & $20(95,2)$ \\
Bone pain & $12(57,1)$ \\
Hepatomegaly & $17(80,9)$ \\
Splenomegaly & $7(33,3)$ \\
Enlarged Lymph Nodes & $3(13,6)$ \\
\hline
\end{tabular}

Fourteen children underwent standard risk national protocol of ALL chemotherapy, and 7 remaining with high risk. Fifteen children were able to complete the induction phase of chemotherapy, the remaining 6 children died before completing. All patients who completed the induction phase of ALL chemotherapy showed remission on bone marrow aspiration evaluation.

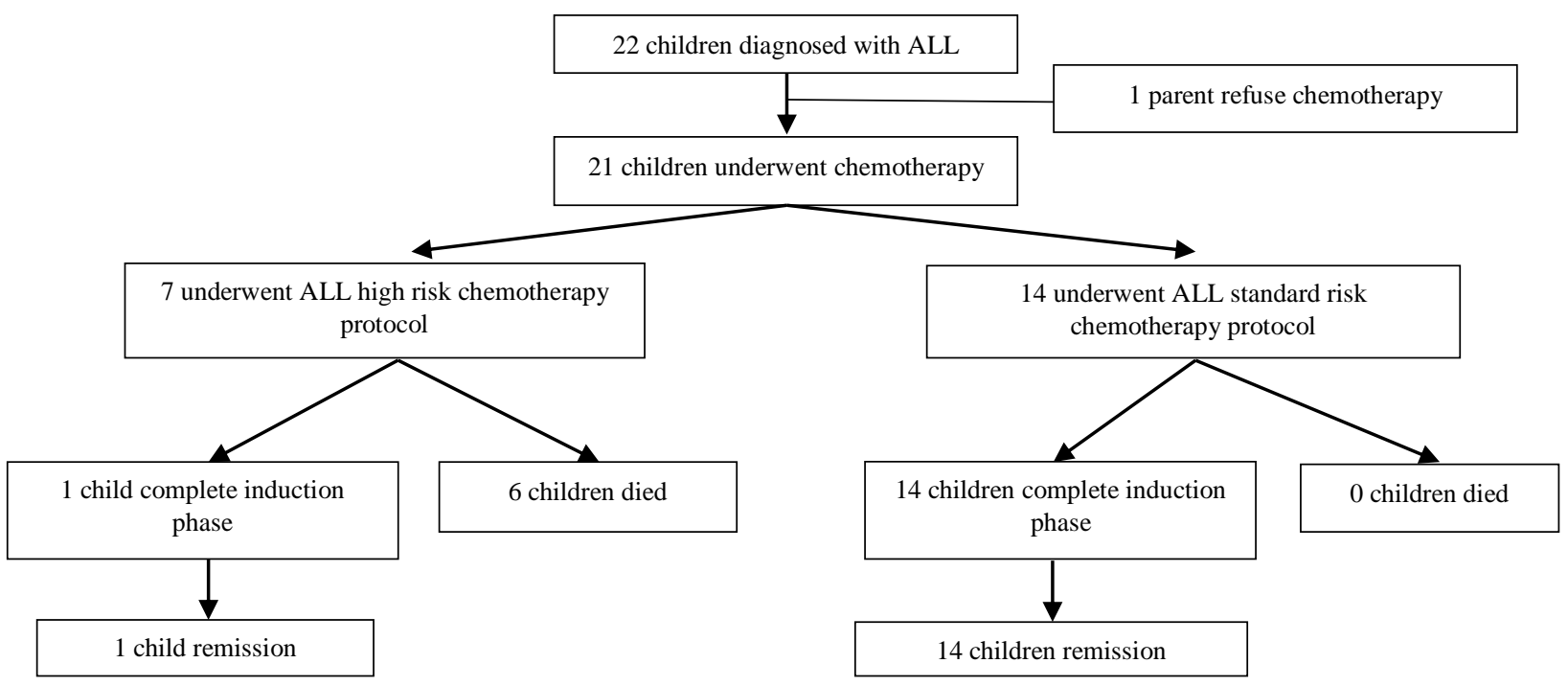

Fig. 1. Flow diagram of study subjects' 


\section{Discussion}

ALL is the most common type of childhood cancer. About $74 \%$ of people under the age of 20 who are diagnosed with leukemia are diagnosed with ALL. Most cases occur between the ages of 2 and 5 years and occur more in boys [4-6]. The results of this study based on age group are in line with a study conducted in Egypt, namely the age group of 1-18 years (77.5\%) more than the age group > 10 years $(22.5 \%)$, boys are more than girls [7]. The same thing was stated by a study in 2009. It stated that the most common cases occurred in the age group 2-10 years (74.2\%), followed by the age group 10-18 years (16.3\%), then 1- 2 years $(9.5 \%)$. Most of them are male with a proportion of $63.9 \%$ and the rest are female with $36.1 \%$ [8].

In this study, most children came with fever complaints, namely 20 children with or without a source of infection, followed by pallor in 16 children, then bone pain in 12 children, while bleeding occurred in 10 children. On physical examination, hepatomegaly was found in 17 children while splenomegaly was only found in 7 children. Enlarged lymph nodes were found in 3 children.

Signs and symptoms of children with ALL vary widely. A study suggested that the most common early signs and symptoms in pediatric ALL patients were pallor, hepato-splenomegaly and thrombocytopenia. This study also stated that only $50 \%$ of their entire sample showed other common signs and symptoms such as fever, fatigue, paleness and bleeding [9].

In this study, morphologically from bone marrow aspiration, ALL L1 predominated in 20 children. On immunophenotyping examination found 13 children categorized as B lymphoid, 4 children as T lymphoid and 4 children read a lymphoid lineage but could not distinguish B cell ALL and T cell ALL. This could happen because $\mathrm{T}$ cell and $\mathrm{B}$ cell markers are equally dominant, so examination with additional markers to determine the type of lymphoid cells is needed.

The classification of leukemia which is still widely used today is based on morphological and cytochemical criteria which were first proposed by the leukemia study group in France, America and England in 1976 which later became known as the French American British (FAB) criteria. The criteria in the classification of ALL based on the FAB classification based on the shape, size of the nucleus and cytoplasm of lymphoblast cells are divided into ALL type L1, type L2, and type L3 [2]. Approximately 85\% of children with ALL have the predominant L1 morphology, 14\% have L2 features, and 1\% have L3 morphology [10].

A recent WHO panel discussion on ALL recommended that the FAB classification be abandoned and advocated the use of an immunophenotyping classification. The 2001 WHO scheme divides cases into B cell precursors, T cells precursors, and ALL mature B cells. The WHO classification was updated in 2008, and has been accepted worldwide based on the recognition of different diseases using a multidisciplinary approach combining morphological, biological and genetic information. become a work nomenclature that has better clinical relevance [10].

In the United States and most European countries, survival rates are around $90 \%$ and $85 \%$, respectively. The five-year survival rate for children 0-14 is 91\%. Meanwhile, the 5-year-survivat rate for children aged 1519 years is $75 \%$. For children who are diagnosed with ALL and remain disease-free after 5 years are generally considered "cured" because acute leukemia rarely recurs after that time. However, in Southeast Asian countries, the highest 5-year survival rate for children aged 0-14 was reported in Malaysia at only $69.4 \%$, followed by Thailand with 55.1\%. Unfavorable results were reported in Indonesia. Studies from Jakarta and Yogyakarta reported 5-year survival rates of $28.9 \%$ and $31.8 \%$, respectively [11].

At Dr Soetomo Hospital in 2013-2014, using the 2013 Indonesian ALL chemotherapy protocol, the outcome of post-induction chemotherapy remission only occurred in $46.4 \%$ of 143 children diagnosed with ALL. Remission of chemotherapy in ALL standard risk is $33.9 \%$ and high risk is $17.8 \%$ with 3 -year free survival rate of $43-56 \%$ [2].

The difference in outcomes between Indonesia and other countries may be due to the high rate of relapse and death during treatment. Several studies reveal that the factors that influence the poor outcome of ALL in 
children in developing countries are inadequate facilities, delayed diagnosis, limited access to health care, neglect of treatment, and suboptimal supportive care [11].

\section{Conclusion}

Indonesian ALL chemotherapy protocol 2018 provide better therapeutic outcomes. Patients who underwent standard risk of Indonesian protocol chemotherapy ALL 2018 have better outcome than high risk. All patient underwent standard risk chemotherapy can complete induction phase. All patients who completed the induction phase showed remission on bone marrow aspiration evaluation.

\section{Acknowledgement}

We wish to thank our staff in the Division of Hematology-Oncology, the doctors, nurses, and the administrator for granting us the permission and necessary support to conduct our research. We did not receive any specific grant for this research from any funding agency in the public, commercial, or not-for-profit sectors. I had full access to all of the data in this study, and I take complete responsibility for the integrity of the data and the accuracy of the data analysis.

\section{Conflict of Interest}

None of the authors disclose any potential conflict of interest.

\section{Ethical Standard}

This study was granted letter of exemption by the Ethic Committee of Dr. Soetomo General Academic Hospital Ref. No.: 0116/LOE/301.4.2/IX/2020. All procedures performed were in accordance with the ethical standards and with the 1964 Helsinki Declaration and its later amendments. Before the subject recruitment, an explanation of general research information was carried out to the subjects and their parents for getting their consent.

\section{References}

[1] Bainer RO, Trendowski R, Pei D, Yang W, Steven W, Goss KH, et al. A p53-regulated apoptotic gene signature predicts treatment response and outcome in pediatric acute lymphoblastic leukemia. Cancer Manag Res 2017;9:397-410.

[2] Larasanti MCS. Perbandingan Stratifikasi Risiko Leulemia Limfoblastik Akut dengan Penambahan Pemeriksaan Imunofenotiping pada Luaran Kemoterapi Indonesian protocol Acute Lymphoblastic Leukemia (ALL) 2013 Fase Induksi di RSUD DR. Soetomo. 2016.

[3] Singh A, Bhatia P, Trehan A, Bansal D, Singh A, Bhatia A. Low spontaneous apoptosis index at diagnosis predicts a high-risk phenotype in paediatric acute lymphoblastic leukaemia. Indian J Med Res 2018;147:248-55. https://doi.org/10.4103/ijmr.IJMR_303_16.

[4] Permono B, Ugrasena IDG. Leukemia akut. Buku Ajar Hematol. Anak, Jakarta: Badan Penerbit IDAI; 2010, p. $236-47$.

[5] Larson RA. Acute lymphoblastic leukemia. William's Hematol., 2016, p. 1505-25.

[6] Horton TM, Steuber CP. Overview of the presentation and diagnosis of acute lymphoblastic leukemia in children and adolescent. UpToDate 2018:17-9.

[7] Abdelmabood S, Fouda AE, Boujettif F, Mansour A. Treatment outcomes of children with acute lymphoblastic leukemia in a middle-income developing country: high mortalities, early relapses, and poor survival. J Pediatr (Rio J) 2020;96:108-16. https://doi.org/10.1016/J.JPED.2018.07.013.

[8] Permatasari E, Windiastuti E, Satari H lrawan. Survival and prognostic factors of childhood acute lymphoblastic leukemia. Paediatr Indones 2009;49:365-71. https://doi.org/10.14238/PI49.6.2009.365-71.

[9] Bernbeck B, Wüller D, Janssen G, Wessalowski R, Göbel U, Schneider D. Symptoms of childhood acute lymphoblastic 
leukemia: red flags to recognize leukemia in daily practice. Klin Padiatr 2009;221:369-73. https://doi.org/10.1055/S-00291239538.

[10] Roganovic J. Acute Lymphoblastic Leukemia in Children. Leukemia, 2013, p. 39-74.

[11] Perdana AB, Saputra F, Aisyi M. Update on Diagnosis of Childhood Acute Lymphoblastic Leukemia (ALL) in Indonesia. Indones J Cancer 2020;14:115-6. https://doi.org/10.33371/IJOC.V14I4.818. 